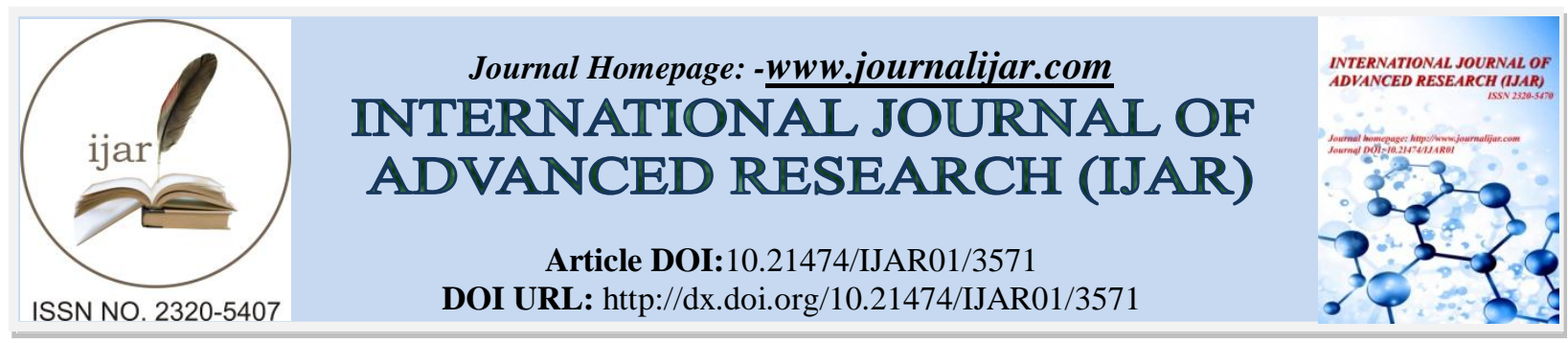

RESEARCH ARTICLE

\title{
CHARACTERIZATION AND ANTIMICROBIAL SUSCEPTIBILITY PATTERN OF CLINICAL ISOLATES OF ENTEROCOCCI AT A TERTIARY CARE HOSPITAL IN BHAVNAGAR, GUJARAT.
}

\author{
Dr. Dhvani D. Parmar.
}

\section{Manuscript Info}

Manuscript History

Received: 03 January 2017

Final Accepted: 07 February 2017

Published: March 2017

Keywords: -

Enterococci, Catalase test, Bile esculin agar, Sugar fermentation test.

\begin{abstract}
Enterococcal infection is a life threatening systemic infection prevalent in developing countries like India and is a major public health problem.Enterococciare important nosocomial pathogen of human disease; second most common cause of nosocomial urinary tract and wound infections; third most common cause of nosocomial bacteremias. Because of their resistance to different antimicrobial agents these bacteria are often involved in serious super-infections among patients receiving broad-spectrum anti-microbial therapy. Correct diagnosis is crucial in proper management of Enterococcal infection, so laboratory tests are essential to establish identification of Enterococcal species. The study was conducted to isolate and characterize Enterococcus species from different clinical samples and to perform antimicrobial susceptibility testing in all age groups and both genders. Out of 200 Enterococcal species isolated, 164 (82\%) were E. faecalis and $34(17 \%)$ were E. faecium; majority were isolated from urine $(60 \%)$ and blood (35.5\%); they showed more resistance to penicillin $(92 \%)$, less resistance to vancomycin $(1 \%)$ and no resistance to linezolid $(0 \%)$.
\end{abstract}

Copy Right, IJAR, 2017, all rights reserved.

\section{Introduction:-}

Enterococci are Gram-positive cocci which are normal residents of the gastrointestinal tract, biliary tract and, in lower numbers, of the vagina and male urethra, in saliva. They are becoming increasingly important nosocomial pathogen of human disease, largely because of their resistance to antimicrobial agents to which other streptococci are generally susceptible.

Enterococci are the second most common cause of nosocomial urinary tract and wound infections and the third most common cause of nosocomial bacteremias. Enterococcus species also cause endocarditis, intraabdominal and pelvic infections, soft-tissue infections, neonatal sepsis and rarely meningitis ${ }^{1}$.

Because of their resistance to penicillin and cephalosporin group, the acquisition of high-level resistance to aminoglycosides, and now the emergence of vancomycin resistance, these bacteria are often involved in serious super-infections among patients receiving broad-spectrum anti-microbial chemotherapy. Newer agents such as linezolid and daptomycin may also be effective; other agents such as chloramphenicol may be used when multidrugresistant strains are encountered. For urinary tract isolates, nitrofurantoin, tetracycline, or quinolone may be effective $\mathrm{e}^{1,2}$. 
In mid-1980s, Enterococci were distinguished from streptococci and related taxa by their ability to grow at $10^{\circ} \mathrm{C}$ and $45^{\circ} \mathrm{C}$, growth in the presence of $6.5 \% \mathrm{NaCl}$, growth at $\mathrm{pH} 9.6$, grow in $0.1 \%$ methylene blue milk, ability to hydrolyze esculin in the presence of $40 \%$ bile, and production of pyrrolidonyl arylamidase (PYR) $)^{1,3}$.

Enterococcus faecalis is the most common isolate found in human clinical specimens, followed by Enterococcus faecium. Other Enterococcal species, including E. avium, E. cassiliflavus, E. cecorum, E. durans, E. gallinarum, E. hirae, E. raffinosus, E. malodoratus, E. dispar and E. mundtii are infrequently isolated from human infections.

Risk factors for the development of bacteremias include advanced age, immunosuppression, underlying disease and conditions (e.g.; prematurity, diabetes, malignancy, congestive heart failure, renal insufficiency, deep-seated infections), prior gastrointestinal, genitourinary, or respiratory tract instrumentation, long-term hospitalization, and the use of broad-spectrum antibiotics having little or no antienterococcal activity (e.g., cephalosporin) ${ }^{1}$.

Isolation measures to prevent person-to-person transmission according to CDC's Guidelines includes ${ }^{4}$ :

- Hand washing with antimicrobial soaps and hand rubs with alcohol-based solutions,

- Wear gloves, Wear a gown,

- Dedicate the use of items such as stethoscopes, blood pressure cuffs, and thermometers to a single patient.

\section{Aims and Objectives:-}

- To isolate Enterococci from different clinical samples and to speciate Enterococci by different tests.

- To determine antibiotic susceptibility pattern of Enterococcus species for proper treatment of patient by specific antimicrobial drugs.

\section{Materials and Methods:-}

The study was conducted from May 2014 to August 2015 in Sir Takhtsinhji General Hospital, Bhavnagar, Gujarat, India.

In this study 200 Enterococci species isolated from different clinical samples- Urine, blood culture, pus, catheter tips and body fluids collected in sterile container from all patients attending to Hospital. Samples were collected from patients having risk factors like advanced age, immunosuppression, prematurity, diabetes, malignancy, congestive heart failure, renal insufficiency, deep-seated infections, prior gastrointestinal, genitourinary, or respiratory tract instrumentation, long-term hospitalization, and long- term use of broad-spectrum antibiotics.

\section{Identification and Characterization:-}

Urine samples were inoculated on CLED agar, other mentioned samples were inoculated on Blood agar. After overnight incubation at $37^{\circ} \mathrm{C}$ colonies were identified. Further identification was done by inoculation on Bile-Esculin agar, PT agar, by doing Gram stain, Catalase test, Growth in $6.5 \% \mathrm{NaCl}$ and Carbohydrate Fermentation Test using six sugars- Mannitol, Sorbitol, Sucrose, Raffinose, Lactose, Arabinose.

\section{Antimicrobial Susceptibility Testing:-}

Antibiotic susceptibility testing of the isolates was performed on Muller Hinton agar by Kirby-Bauer disc diffusion method $^{5}$. The antibiotic concentration of disc used and zone size interpretation was accordance to Clinical Laboratory Standards Institute (CLSI) ${ }^{6}$. These antimicrobial drugs are Ampicillin, Ampicillin-Sulbactum, Penicillin, Piperacillin-Tazobactum, Linezolid, Ciprofloxacin, Tetracycline, Roxithromycin, Vancomycin, High level Gentamycin $(120 \mu \mathrm{g})$, High level Streptomycin $(300 \mu \mathrm{g})$, and Nitrofurantoin. Commercially available six mm disks (Himedia Laboratories, Mumbai) were used.

\section{Quality Controls:-}

Standard operative procedures were followed during processing of each sample. E.faecalis VRE ATCC 51299and E.faecalis VSE ATCC 29212 were used as a reference strains ${ }^{7,8}$.

\section{Results:-}

Out of 1850 culture positive samples proceeded, 200 Enterococci were isolated from urine, blood, pus, catheter tip (hospital acquired) \& body fluid (peritoneal fluid), suggesting 11\% prevalence rate of Enterococci. 
Enterococci are Gram positive oval cocci, arranged in pairs at an angle to each other; catalase negative; can grow in $6.5 \% \mathrm{NaCl}$. After overnight incubation small yellow or pink colonies were formed on CLED medium; small, cream or white or grayish-white, smooth entire, non-hemolytic colonies were formed on Blood agar. As Enterococci hydrolases esculin in presence of $40 \%$ bile, blackening on BEA media seen. E.faecalis could grow on PT agar producing black colonies, but E.faecium could not ${ }^{1,3,9}$.

Isolated Enterococci species distribution, sample wise, age and sex wise distribution of Enterococci species shown in Table - 1, 2, 3 and 4 respectively.

Table 1:- Species of Enterococci isolated

\begin{tabular}{|c|c|c|}
\hline Enterococci Species & Number & Percentage \\
\hline E.faecalis & 164 & $82 \%$ \\
\hline E.faecium & 34 & $17 \%$ \\
\hline Other & 2 & $1 \%$ \\
\hline
\end{tabular}

Other Enterococcal species might be E.avium, E.gallinarum, E.cassiliflavus, E.durans, E.raffinosus or E.mundtii.

Table 2:-Samples showing Enterococcal species isolation

\begin{tabular}{|c|c|c|}
\hline Samples & Number & Percentage \\
\hline Urine & 120 & $30 \%$ \\
\hline Blood & 71 & $1.5 \%$ \\
\hline Pus & 3 & $1 \%$ \\
\hline Peritoneal fluid & 2 & $2 \%$ \\
\hline Catheter tip & 4 & \multicolumn{2}{|c|}{} \\
\hline
\end{tabular}

Table 3:- Age-wise distribution of Enterococci Species.

\begin{tabular}{|c|c|c|}
\hline Age Group & Number & Percentage \\
\hline <1 year & 44 & $22 \%$ \\
\hline 1-15 year & 32 & $16 \%$ \\
\hline 16-30 year & 28 & $14 \%$ \\
\hline 31-45 year & 40 & $20 \%$ \\
\hline 46-60 year & 34 & $17 \%$ \\
\hline$>60$ year & 22 & $11 \%$ \\
\hline
\end{tabular}

Table 4:- Sex-wise distribution of Enterococci Species.

\begin{tabular}{|c|c|c|}
\hline Sex & Number & Percentage \\
\hline Female & 113 & $56.50 \%$ \\
\hline Male & 87 & $43.50 \%$ \\
\hline
\end{tabular}

The antimicrobial susceptibility pattern ofEnterococcispecies has been shown in Table-5. In spite of predominance of E.faecalis, it was observed that E.faecium was more resistant than E.faecalis.

Table 5:-Antimicrobial drug resistance in Enterococci.

\begin{tabular}{|c|c|c|}
\hline Antimicrobial Drugs & $\begin{array}{c}\text { Isolates Resistant to Antimicrobial Drugs } \\
\text { (200 species) }\end{array}$ & Percentage of Resistance \\
\hline Ampicillin & 106 & $53 \%$ \\
\hline Ampicillin-Sulbactum & 83 & $41.5 \%$ \\
\hline Penicillin & 184 & $52 \%$ \\
\hline Piperacillin-Tazobactum & 108 & $0 \%$ \\
\hline Linezolid & 0 & $57.50 \%$ \\
\hline Ciprofloxacin & 115 & $68 \%$ \\
\hline Tetracycline & 136 & $62.50 \%$ \\
\hline Roxithromycin & 125 & $1 \%$ \\
\hline Vancomycin & 2 & $42.50 \%$ \\
\hline High level Gentamycin & 85 & $59 \%$ \\
\hline High level Streptomycin & 118 & $38 \%$ \\
\hline Nitrofurantoin & 76 & \\
\hline
\end{tabular}


Results of Sugar Fermentation Test done on Enterococci species has been shown in Table-6. Acid production occurs from sugar fermentation \& yellowish color develop.

Table 6:- Sugar Fermentation by Species of Enterococci isolated.

\begin{tabular}{|c|c|c|}
\hline Sugars used for fermentation test & E.faecalis & E.faecium \\
\hline Mannitol & + & + \\
\hline Sucrose & + & + \\
\hline Sorbitol & + & - \\
\hline Lactose & + & + \\
\hline Raffinose & - & $+/-$ \\
\hline Arabinose & - & + \\
\hline
\end{tabular}

\section{Discussion:-}

Enterococci colonize the bowels of more than $90 \%$ of healthy humans. E. faecalis accounts for more than $80 \%$ of the Enterococcal isolates in clinical samples, but in recent years E. faecium has become more common, probably because of its greater antibiotic resistance to multiple antibiotics. For the last two decades, Enterococcus have been the $3^{\text {rd }}$ most common cause of hospital-acquired infections (HAI) after E.coli and Staphylococcus aureus and ahead of Pseudomonas aeruginosa. The most common nosocomial infections caused by Enterococci are urinary tract infections (associated with instrumentation and antimicrobial administration) followed by intra-abdominal and pelvic infections. They also cause endocarditis, neonatal sepsis and rarely meningitis.

In last two decades infections due to Enterococci have increased. Now, it is among the leading causes of bacteremias. Enterococci is the second most common cause of surgical infections and the third most frequently reported cause of HAIs. Gastrointestinal colonization, length of stay in hospital, ICU stay, older age, neutropenia, hematological malignancies and transplantation are risk factors identified for Enterococcal colonization and infection $^{11}$.

The emergence of high-level resistance to aminoglycosides in Enterococci, particularly E.faecalis and E.faecium, seriously affected the therapeutic approach; vancomycin become a first-line drug effective against Enterococci ${ }^{12}$.

Until the early 1980s, the susceptibility of Enterococci to ampicillin and vancomycin remained fairly predictable. Since that time, Enterococcus species resistant to Ampicillin and vancomycin have been reported with increasing frequency. Linezolid seemed to be an appropriate therapeutic option for VRE in our study ${ }^{13}$.

In present study isolated E.faecalis and E.faecium were $82 \%$ and $17 \%$, which is comparable to studies conducted by Bose et $\mathrm{al}^{14}$ (82\% and $18 \%$ ) and Gupta et $\mathrm{al}^{15}$ (80\% and $\left.20 \%\right)$; In present study only two other species (1\%) isolated which might be E. durans, E. raffinosus, E. avium, E. gallinarum comparable to study done by Grey et $\mathrm{al}^{16}(1.2 \%)$.

In present study, the most common clinical sample from which Enterococci were isolated was urine (60\%), comparable tostudies conducted by Agrawal et $\mathrm{al}^{17}(62.79 \%)$ and Bhatt et al ${ }^{18}(57 \%)$; followed by blood (35.50\%), pus $(1.50 \%)$, catheter tips $(2 \%)$, peritoneal fluid $(1 \%)$.

Present study showed that isolation of Enterococci from all age groups, ranging from newborn baby to an old age of 86 years and highest percentage $(22 \%)$ of Enterococcal isolates was obtained from <1 year age group, similar to observed by Nepal et al ${ }^{19}(20 \%)$.

In present study female predominance $(56.5 \%)$ over males seen corresponds to the Bhatt et al ${ }^{18}(54 \%)$. Greater vulnerability to acquire urinary tract infection and pelvic infection due to anatomic position of urethra and genital tract might have played a role for predominant female infection by this organism ${ }^{11,18}$.

In present study, beta-lactam drugs, flouroquinolones, high level aminoglycosides and tetracycline were found to be highly resistant; penicillin was most resistant $(92 \%)$, comparable to study done by Bhatt et $\mathrm{al}^{18}(97.5 \%)$. In present study linezolid has shown $0 \%$ resistance, followed by vancomycin shown $1 \%$ resistance, comparable to studies conducted by Bose et $\mathrm{al}^{14}$ (linezolid $0 \%$ resistant) and Saraswathy et $\mathrm{al}^{20}$ (vancomycin $1 \%$ resistant). 


\section{Conclusion:-}

- In developing country like INDIA Enterococciare important nosocomial pathogens of human disease; because of their resistance to antimicrobial agents like penicillins, cephalosporins group, the acquisition of high-level resistance to aminoglycosides and now the emergence of vancomycin resistance, these bacteria are often involved in serious superinfections among patients receiving broad-spectrum anti-microbial chemotherapy. Prompt diagnosis is essential for the patient and the community.

- Identification of Enterococci done by urine culture, blood culture, Gram stain and also by other methods like catalase test negative, its ability to grow at $10^{\circ} \mathrm{C}$ and $45^{\circ} \mathrm{C}$, growth in the presence of $6.5 \% \mathrm{NaCl}$, ability to hydrolyze esculin in the presence of $40 \%$ bile and sugar fermentation test.

- Mainly two species of Enterococci were isolated, E. faecalis (82\%) \&E. faecium (17\%).

- Out of 200 Enterococcal species $60 \%$ were isolated from urine samples, followed by $35.5 \%$ were from blood samples.

- As far as antibiotic susceptibility was concerned, resistance to penicillin (92\%), suggest that resistance to $\beta$-lactam group was more among Enterococcus species. Resistance to vancomycin shown in $1 \%$ Enterococcal species, linezolid is more effective drug as all isolates are sensitive to it.

\section{References:-}

1. Koneman EW, Allen SD, Janda WM, Schreckenberger PC, Winn WC. Color Atlas and Textbook of Diagnostic Microbiology. $6^{\text {th }}$ ed. Philadelphia: Lippincott; 2006; 700-705.

2. Forbes BA, Sahm DF, Weissfeld AS. Tille PM. Bailey and Scott's Diagnostic microbiology. $13^{\text {th }}$ ed. Mosby: Elsevier; 2014.

3. Ananthanarayan R, Paniker CKJ. Textbook of Microbiology. Hyderabad; Orient Blackswan Private Limited; 2013; $9^{\text {th }}$ ed : 217-218.

4. Susan L Fraser, MD, Robert A Salata, MD, Curtic J Donskey, MD. Enterococcal Infections. Medscape Updated, June 2015.

5. Mulla et al. Prevalence of Enterococci with higher resistance level in a tertiary care hospital: A matter of concern. National Journal of Medical Research. 2012, vol 2, issue 1, p: 25-27.

6. Performance standards for antimicrobial susceptibility testing. CLSI twenty first informational supplement M100S21. 2011; 31(1).

7. Rajkumari et al. Magnitude of Enterococcal bacteria in trauma patients admitted from intensive trauma care; A tertiary care experience from South Asian country. J of Lab Physician. 2015, vol 7, issue 1, p: 38-42.

8. Sarita et al. Antibiotic resistance in fecal Enterococcal in hospitalized patients. IJPM. 2010, 53(4), 898-899.

9. Monika Cheesbrough. District Laboratory Practice in Tropical Countries. $2^{\text {nd }}$ edition, update, part-2. Cambridge, New Delhi, 2012, p-69,165.

10. PJ Desai, D Pandit, M Mathur, A Gogate. Prevalence, identification and distribution of various species of enterococci isolated from clinical specimens with special reference to urinary tract infection in catheterized patients. IJMM, Year: 2001, Volume : 19, Issue : 3, Page : 132-137

11. Aher CS et al. Vancomycin resistant Enterococci, An emerging threat. Int. J. Curr. Microbiol. App. Scie. 2014 , vol 3 (2), 14-19.

12. Jawetz, Melnick, Adelberg. Medical Microbiology; $24^{\text {th }}$ ed, Mc Graw Hill, 2007, 254-259.

13. Vidyalakshmi PR,Gopala Krishnan R, RamasubramanianV et al. Clinical, Epidemiological, and Microbiological Profile of Patients with Vancomycin-Resistant Enterococci from a Tertiary Care Hospital. J Glob Infect Dis. 2012 Apr-Jun; 4(2): 137-138.

14. Bose S, Ghosh AK, Barapatre R et al. Prevalence of drug resistance among Enterococcus spp isolated from a Tertiary Care Hospital. International Journal of Medical and Health Sciences, ISSN 2277-4505.

15. Gupta et al. Antimicrobial susceptibility pattern of vancomycin resistant Enterococci to newer antimicrobial agents. Indian J. Med. Res. 141, 2015, 483-486.

16. Grey JW, David S, Pedler SJ. Species identification and antimicrobial susceptibility testing of Enterococci isolated from hospitalized patients. 1991, vol 35, no 9, 1943-1945.

17. Agrawal J, Rajkumar K, Singh M. High level Aminoglycoside resistance and $\beta$-lactamase production in Enterococci at a tertiary care hospital in India. Jpn. J. Infec. Dis, 2009, 62, 158-159.

18. Bhatt MP, Patel A, Sahni AK, et al. Emergence of multidrug resistant enterococci at a tertiary care center. Med Journal ARMED India, 71 (2015) 139-144.

19. HP Nepal, B Khanal, A Acharya et al. Characterization and antimicrobial resistance of clinical isolates of enterococci in tertiary care hospital in eastern Nepal. Health Renaissance 2013; Vol. 11 No. 1; 17-22.

20. Saraswathy P, Karunakaran S, Narayanan S. Antimicrobial resistance profile and characterization of Enterococcus species from various clinical sample in a tertiary care hospital. Int. J. of Med. Res. Health Scie. 2013, vol 2 (3), 328333. 